CASE REPORT

\author{
Y.J. Jin \\ S.K. Chung \\ O.K. Kwon \\ H.J. Kim
}

\section{Spinal Intraosseous Arteriovenous Fistula in the Fractured Vertebral Body}

SUMMARY: A 68-year-old woman presenting with progressive paraparesis was diagnosed with an AVF involving a previously fractured L1 vertebral body on which feeders from multiple segmental arteries converged. The most distinctive feature in our patient was that the fistula was located in the vertebral body. Transarterial embolization of the segmental arteries with coils and glue resulted in total obliteration of the fistula, which in turn resulted in symptom improvement.

ABBREVIATION: AVF = arteriovenous fistula

$\mathbf{E}$ pidural or paraspinal AVF is very rare, and few cases have been reported in the literature. ${ }^{1-4}$ It is not a well-known entity, and it is not always easy to distinguish an epidural AVF from a dural AVF when reflux into the perimedullary veins is present. Although intradural reflux is rare in paravertebral shunts, it may show angiographic findings similar to those of a dural AVF and venous hypertension of the spinal cord. ${ }^{2} \mathrm{We}$ report a case of trauma-related intraosseous fistula directly draining through the L1 basivertebral vein into the epidural, paraspinal, and perimedullary venous plexuses.

\section{Case Report}

A 68-year-old woman presented with a 5-month history of a tingling sensation in both legs and a 2-month history of gradually progressive paraparesis. In addition, she also had a 9-year history of back pain after falling from a ladder.

Neurologic examination revealed that she was unable to walk steadily and had paraparesis (grade IV/V). Sensory examination demonstrated paresthesia and hypesthesia of the perianal area and both lower extremities, symmetrically. Urinary retention and fecal incontinence were noted. Her deep tendon reflexes were slightly increased in the bilateral lower extremities. Pathologic reflexes, such as ankle clonus and Babinski sign, were positive.

Engorged intraosseous and perimedullary veins accompanying cord signal-intensity changes were detected on CT scans (Fig 1A, - B) and MR images (Fig 2). Spinal angiography showed intraosseous fistulas and multiple feeders from bilateral L1 and L2 and right T12 segmental arteries (Fig 3).

She was diagnosed with trauma-related epidural or paraspinal AVF. We confirmed that the venous flow drained mainly via the intraosseous L1 basivertebral vein into the epidural venous plexus on the left in a downward direction; then, the flow drained into the left paravertebral veins on the spinal angiography. We tried embolization through the left femoral vein but failed to find the connection between the epidural or paravertebral vein and the left femoral vein or inferior vena cava. Transarterial embolization was selected as the treatment technique.

\section{Received September 3, 2008; accepted after revision June 15, 2009}

From the Department of Neurosurgery, Seoul National University College of Medicine, Seoul, South Korea; and Clinical Neuroscience Center, Seoul National University Bundang Hospital, Seongnam, South Korea.

Please address correspondence to Sang Ki Chung, MD, PhD, Seoul National University College of Medicine, Clinical Neuroscience Center, Seoul Nation University Bundang Hospital, 300 Gumi-dong, Bundang-gu, Seongnam-si, Gyeonggi-do 463-707, South Korea; e-mail: skcsnubh@gmail.com

DOI 10.3174/ajnr.A1780
The muscular branches in each segmental artery were protected by coils due to the delay of wound healing, which might have been caused by occlusion of superficial arteries during embolization. Multiple feeders were occluded, including the venous sac, with $33 \%$ glue-that is, a mixture of $n$-butyl cyanoacrylate (Histoacryl; B. Braunn, Melsungen, Germany) and iodized oil (Lipiodol; Guerbet, Aulnay-Sous-Bois, France) at a ratio of 1:2. The exact volume of embolic material was not calculated. The final angiography confirmed total obliteration of the fistula.

Neurologic examination performed 5 days after surgery revealed that the patient's leg weakness had improved $\left(\mathrm{Grade} \mathrm{IV}^{+} / \mathrm{V}\right)$ and that her perianal hypesthesia and urinary retention had resolved. Follow-up MR images obtained 5 days after the intervention showed a reduction in cord signal intensity, near obliteration of perimedullary signal-intensity voids at the thoracolumbar junction, and thrombotic change in the basivertebral and epidural veins. MR images obtained 7 months later showed the disappearance of cord signal-intensity change and enlarged pial veins. The patient was able to walk without assistance, but she continued to experience a mild residual tingling sensation in the perianal area and in both legs. She regained full control of sphincter function.

\section{Discussion}

In the present study, we report a unique case of AVF occurring after an L1 compression fracture. There were many interesting findings in this case. The most peculiar finding was that the fistula was located in the vertebral body. It has been hypothesized that trauma can lead to the development of an epidural or paraspinal AVF. There have been some case reports of epidural shunts related to trauma, but definite evidence of vertebral injury, such as a vertebral fracture, was seldom observed on radiologic examination. ${ }^{1,3,4}$ Several mechanisms of fistula formation have been suggested. Trauma, with or without vertebral fracture, results in microtears of the affected arterial wall and produces an AVF. ${ }^{5}$ It is also possible that traumatic events can cause thrombosis or thrombophlebitis of the veins. A fistula can develop due to arterial growth during the process of organization and recanalization. ${ }^{5,6}$ Another mechanism of fistula formation is increased venous pressure due to impaired venous drainage after trauma causing spontaneous occurrence. ${ }^{6}$

There is no question that trauma was the cause of fistula formation in our patient. AVFs were located in the vertebral body near the surface. The main feeder was the right L1 segmental artery with a high-flow shunt. The shunt was con- 

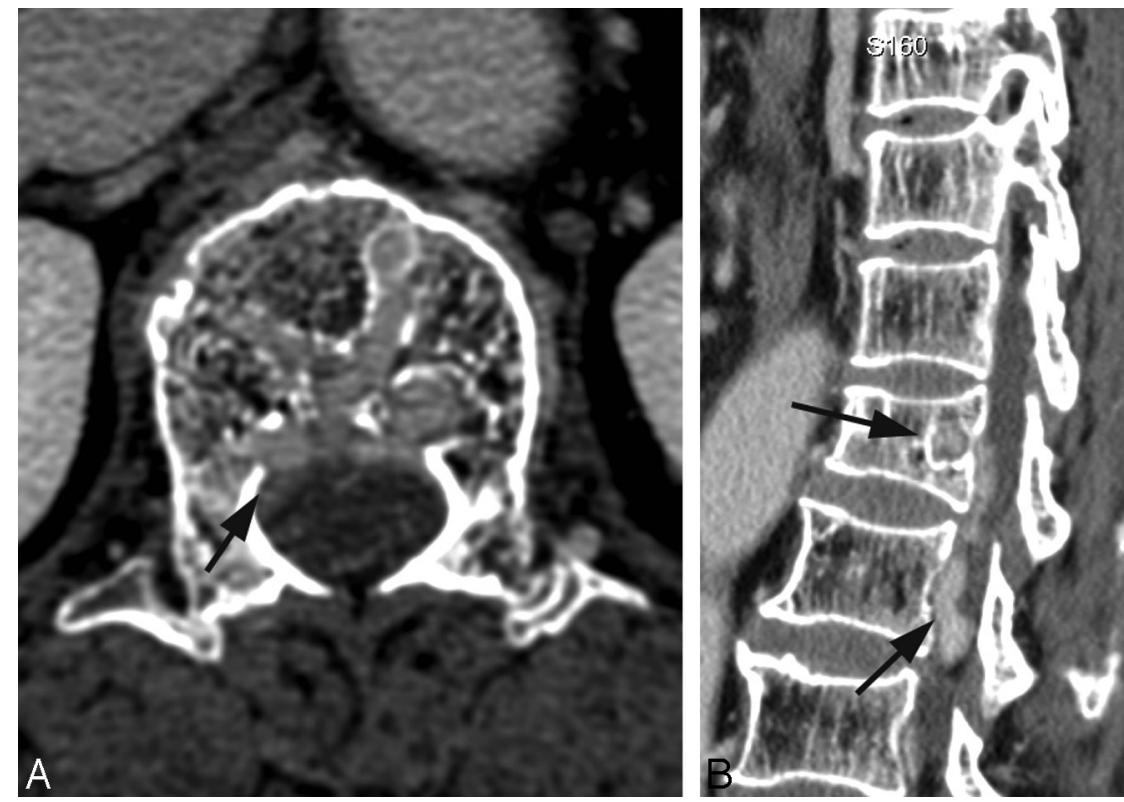

Fig 1. $A$ and $B$, Enhanced CT scans show the old L1 compression fracture, the erosion of bony trabeculae caused by dilation of the basivertebral vein (arrow), and longitudinally enlarged epidural veins (arrows).
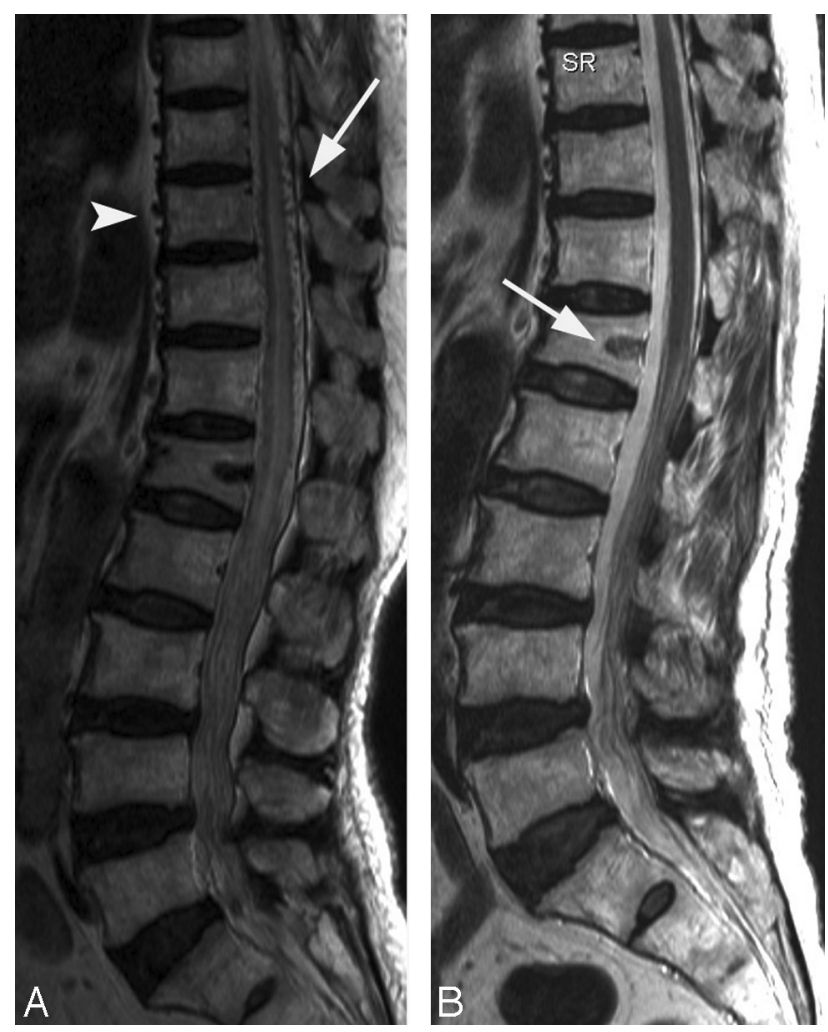

Fig 2. A, Preoperative sagittal T2-weighted MR image demonstrates high signal intensity and edematous change of the spinal cord from $T 7$ to the conus. Especially, the engorged $\mathrm{L} 1$ basivertebral vein gives rise to the bony erosion in the marrow. Engorged perimedullary veins are found anterior and posterior to the spinal cord from C3 to L5 (arrow). No intramedullary nidus could be detected. Dilated paravertebral veins are also seen anterior to the vertebral body from the level of T6 to $L 1$ (arrowhead). B, Postoperative sagittal image shows thrombosed basivertebral veins at $\mathrm{L} 1$ (arrow). There is no evidence of recanalization. Perimedullary veins and cord signal intensity changes are not visible.

nected to the basivertebral vein and formed a large defect in the trabecular bone. We hypothesize that the bone defect might have originated from the fracture line that was made at the time of trauma. It is possible that the high-pressure blood flow might have widened the defect as time passed.

Suh et $\mathrm{al}^{4}$ reported 2 cases of osseous epidural AVFs as a variant of a paraspinal or epidural AVF. They differentiated the dilated venous sac that caused the bony defect from dilated venous ectasia of Rendu-Osler-Weber disease. The latter did not receive arterial blood supply directly, and the fistula was not located in the epidural space. They presumed that the osseous epidural AVF was located near the bony margin and recruited multiple feeders from the dura and bone. On the basis of the ideas of Suh et al, our case is one of osseous epidural AVF, and we believe that it may be a representative case because the shunt surgery occurred throughout the entire vertebral body. The venous sac only involved a small portion of the vertebra in the cases reported by Suh et al.

An epidural AVF can cause neurologic symptoms in several ways. A direct connection between an extradural artery and vein leads to the development of a high-flow fistula, engorgement of the epidural venous system, and compression of the spinal cord by dilated veins or an epidural hematoma. The high pressure in the epidural venous system may induce intradural venous hypertension, and the shunt surgery of a large volume of arterial blood into the venous system can take blood from the spinal cord. Direct compression of the spinal cord or nerve root by dilating epidural veins or possible epidural hematoma can cause myelopathy or radiculopathy. Intradural reflux in an epidural AVF, as seen in this patient, is rare, and only a few cases have been reported. ${ }^{1,2}$ The engorged intradural veins and prominent signal-intensity changes in the spinal cord make it challenging to differentiate an epidural AVF with intradural reflux from a dural AVF. The long duration from the initial trauma until the onset of myelopathy may be related to the location of the fistula. Because the fistulas were located in the vertebral body, it may have taken a long time to form a shunt surgery fistula that was large enough to cause myelopathy.

Endovascular surgery was the most reasonable treatment 

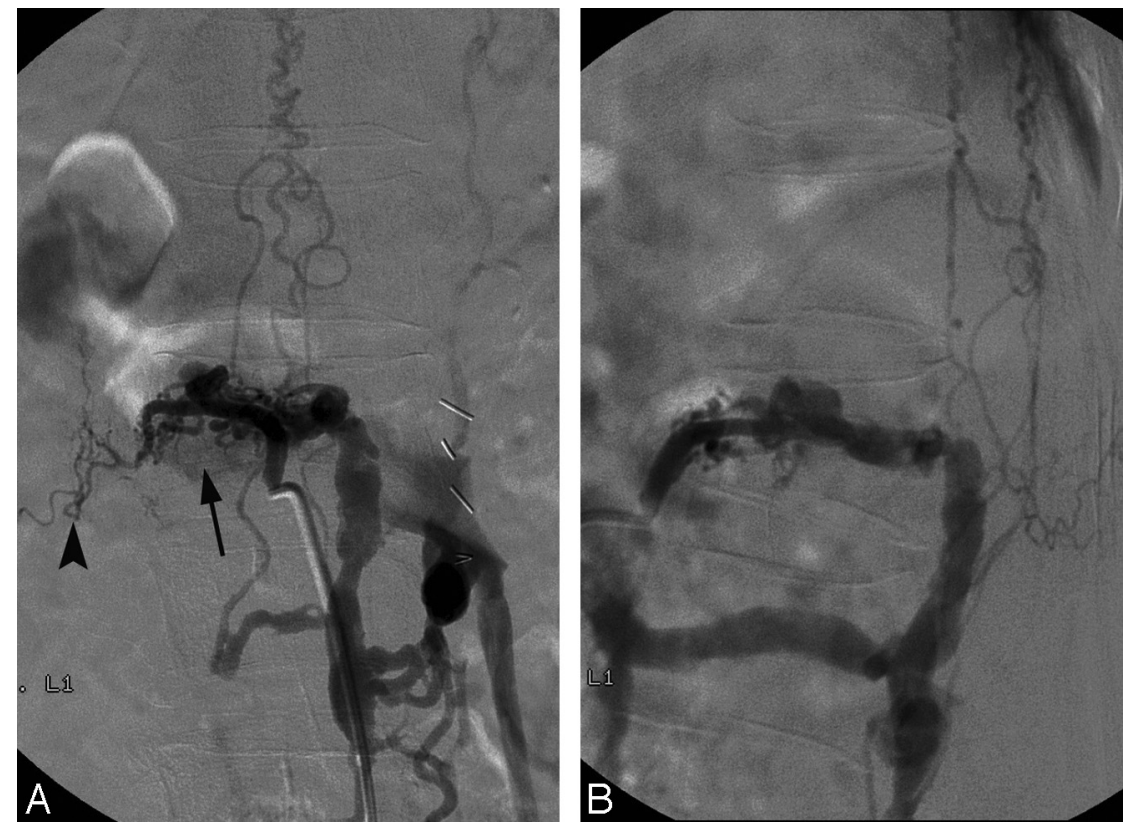

Fig 3. A, Spinal angiogram reveals a high-flow intraosseous AVF fed by multiple fine branches of segmental arteries from T12 to $L 2$ on the right side and from $L 1$ to $L 2$ on the left side There are multiple fistulas between the right L1 segmental arterial wall and the L1 basivertebral vein in the fractured L1 vertebral body (arrow). The right $\mathrm{L} 1$ segmental artery is the main feeder with a high-flow shunt, and the right T12 segmental artery is the second feeder. The other feeders are low-flow shunts. The muscular branch of the L1 segmental artery is stenotic, and the distal arterial flow is more tenuous than the others (arrowhead). B. The venous flow drains mainly via the intraosseous L1 basivertebral vein into the epidural venous plexus on the left in a downward direction. The ascending retrograde drainage into the perimedullary veins anterior and posterior to the spinal cord is noted on both sides at the level of the L2 body.

technique for this patient. All feeders were accessible. Surgery required extensive exposure of the spinal column. Suh et al $^{4}$ recommended the transvenous approach for effective embolization of multiple feeders, and though we agree with their recommendation, the venous approach was not possible in this case.

\section{Conclusions}

We present a rare case of posttraumatic osseous AVF draining directly through the L1 basivertebral vein, with a reflux to the epidural, paraspinal, and perimedullary venous plexuses. Endovascular therapy via a transarterial approach was an effective and minimally invasive treatment technique.

\section{References}

1. Cognard C, Semaan H, Backchine S, et al. Paraspinal arteriovenous fistula with perimedullary venous drainage. AJNR Am J Neuroradiol 1995;16: 2044-48

2. Pirouzmand F, Wallace MC, Willinsky R. Spinal epidural arteriovenous fistula with intramedullary reflux. J Neurosurg 1997;87:633-35

3. Heuer GG, Gabel BC, Bhowmick DA, et al. Symptomatic high-flow arteriovenous fistula after a C2 fracture: case report. J Neurosurg Spine 2008;8: 381-84

4. Suh DC, Choi CG, Sung KB, et al. Spinal osseous epidural arteriovenous fistula with multiple small arterial feeders converging to a round fistular nidus as a target of venous approach. AJNR Am J Neuroradiol 2004:25:69-73

5. McCutcheon IE, Doppman JL, Oldfield EH. Microvascular anatomy of the dural arteriovenous abnormalities of the spine: a microangiographic study. J Neurosurg 1996;84:215-20

6. Ushikoshi S, Goto K, Uda K, et al. Vertebral arteriovenous fistula that developed in the same place as a previous ruptured aneurysm: a case report. Surg Neurol 1999;51:168-73 